\title{
The Multilayered Innate Immune Defense of the Gut
}

\author{
Laure El Chamy ${ }^{1}$, Nicolas Matt ${ }^{2}$, Monde Ntwasa ${ }^{3}$, Jean-Marc Reichhart ${ }^{2}$
}

In the wild, the fruit fly Drosophila melanogaster thrives on rotten fruit. The digestive tract maintains a powerful gut immune barrier to regulate the ingested microbiota, including entomopathogenic bacteria. This gut immune barrier includes a chitinous peritrophic matrix that isolates the gut contents from the epithelial cells. In addition, the epithelial cells are tightly sealed by septate junctions and can mount an inducible immune response. This local response can be activated by invasive bacteria, or triggered by commensal bacteria in the gut lumen. As with chronic inflammation in mammals, constitutive activation of the gut innate immune response is detrimental to the health of flies. Accordingly, the Drosophila gut innate immune response is tightly regulated to maintain the

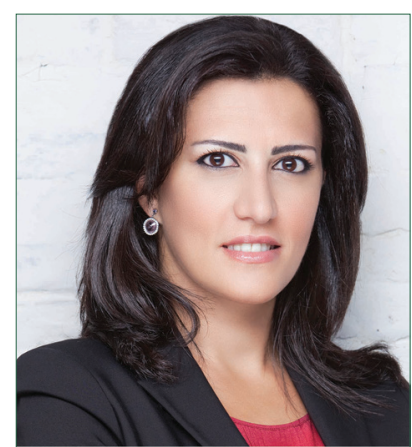

Dr. Laure El Chamy endogenous microbiota, while preventing infections by pathogenic microorganisms. (Biomed J 2015;38:276-284)

\section{Key words: digestive tract, Drosophila, innate immune response}

$\mathrm{T}^{\mathrm{s}}$ The optimal functioning of the intestinal immune response requires discrimination between the indigenous, symbiotic gut microbiota and potential microbial pathogens. Understanding of the antimicrobial responses in the Drosophila gut has been advanced by the development of oral infection models, ${ }^{[1-8]}$ studies of gut structure, ${ }^{[3,9-20]}$ characterization of commensal microbiota, ${ }^{[6,21-26]}$ and use of axenic flies. ${ }^{[27-30]}$ These studies reveal that the basal immune response is weakly induced by the commensal gut microbes and strongly enhanced upon pathogen immune challenge. ${ }^{[23]}$ Regulatory mechanisms which dampen the basal immune response are essential to avoid chronic lethal reactions, ${ }^{[31]}$ but the system must remain responsive to acute infectious challenges. ${ }^{[29,32]}$ Ingestion of pathogenic bacteria causes physiopathological alterations in epithelial barriers, both from bacterial toxins and by damage resulting from the host's own immune response..$^{[3,5,31,33-36]}$ Subsequent repair mechanisms promote the regeneration of stem cells to re-establish gut integrity and a homeostatic balance at the intestinal barrier. These repair mechanisms involve several host signaling pathways. ${ }^{[29,32,37-40]}$ In addition, re- generation of intestinal cells is also modulated indirectly by the commensal microbiota, which complements the host physiology in several ways. ${ }^{[29,34,41,42]}$ Notably, the commensal microbiota seem to be involved in the regulation of larval growth and have been linked to age-related diseases, associated with gut dysplasia and loss of intestinal integrity. ${ }^{[43-47]}$ Finally, as in mammals, commensal microbes seem to contribute to protective reactions against pathogen challenge. ${ }^{\left[{ }^{848]}\right.}$ Study of gastrointestinal infections by the yeast Candida albicans in Drosophila larvae indicate that the commensal microbes enhance host survival during infection, both in wild-type and immunodeficient flies. Thus, the microbiota may affect pathogens either through direct competition or by altering the gut environment. ${ }^{[8,17]}$ In this review, we provide the latest updates on the anatomy of the Drosophila gut and the regulation of the induced immune response in the intestinal mucosa. The repair mechanisms of the injured intestine are beyond the scope of this article, but recent reviews covering this topic are presented. The role of the commensal microbiota in fly physiology is covered by Ma et al. in this issue.

From the ${ }^{1}$ Laboratory of Drosophila Genetics and Microbial Virulence, EGFEM, Faculty of Sciences, Saint-Joseph University, Beirut, Lebanon; ${ }^{2}$ UPR9022 du CNRS, Institute of Molecular and Cellular Biology, University of Strasbourg, Strasbourg, France; ${ }^{3}$ University of the Witwatersrand, Johannesburg, South Africa

Received: Jan. 19, 2015; Accepted: May 26, 2015

Correspondence to: Dr. Laure El Chamy, Laboratory of Drosophila Genetics and Microbial Virulence, EGFEM, Faculty of Sciences, Saint Joseph University, Beirut, Lebanon. Damas Road, BP 17-5208 - Mar Mikhaël Beirut - 1104 2020, Lebanon. Tel: 961-1-421000 ext. 3485;

E-mail: Laure.chamy@usj.edu.lb

DOI: $10.4103 / 2319-4170.158621$ 


\section{Hold it tight: The Drosophila gut anatomy}

The gut is subdivided into foregut, midgut, and hindgut regions [Figure 1], each consisting of a monolayered epithelium surrounded by visceral muscles, nerves, and tracheae, with the nervous system playing a key role in the regulation of nutrient intake, and fluid and ion balance. ${ }^{[49]}$ Ingested food passes the pharynx and is either stored to the crop or sent directly to the midgut where the digestion starts. ${ }^{[50]}$ The crop itself is a specialized region of the foregut where a variety of immune proteins are secreted, including thioester containing proteins (TEPs). ${ }^{[51]}$ The nature and arrangement of the gut epithelial cells differs depending on their position along the anteroposterior axis and their developmental origin.

The foregut and hindgut epithelia are of ectodermal origin, are lined on the apical side by an impermeable cuticle, and have pleated septate junctions which seal their paracellular spaces. In contrast, the midgut epithelium is of endodermal origin, is not covered by cuticle, and its integrity relies on smooth septate junctions. The midgut is protected by the peritrophic matrix (PM), a semi-permeable membrane allowing the passage of monomeric enzymes and nutrients, but not bacteria: It is here that food absorption occurs. The midgut can be subdivided into an anterior midgut region, the acidified copper cell region, and the posterior midgut, with the copper cell region perhaps functioning as a stomach [Figure 1]. Recently, the adult midgut was further subdivided into six anatomical regions (R0-R5) separated by narrow epithelial boundaries and with distinct metabolic and digestive functions. ${ }^{[40]}$ In the ileum and rectum of the hindgut, water and salt re-absorption takes place from the food bolus. The gut harbors a simple bacterial community, of four or five major phylotypes, from Lactobacillus and Acetobacter genera. ${ }^{[22]}$

In larvae, processing of complex substrates starts before ingestion itself, with clusters of larvae regurgitating digestive enzymes and possibly antimicrobial peptides (AMPs). ${ }^{[52]}$ Four gastric cecae in the anterior midgut represent a specific

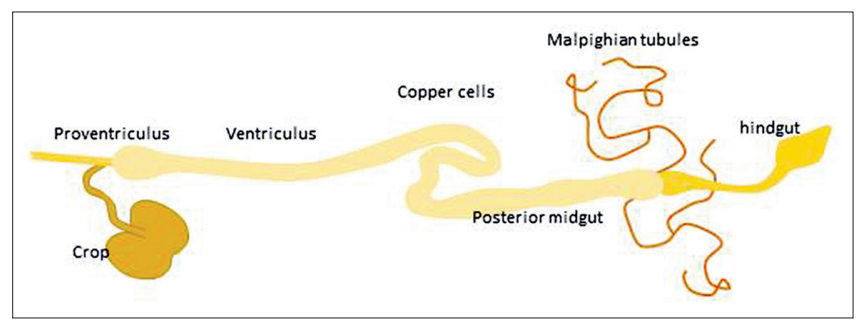

Figure 1: The Drosophila gut anatomy. The Drosophila gut includes three main parts: the foregut (crop), the midgut, and the hindgut. The midgut is divided in four sections: the proventriculus, the ventriculus, the copper cells, and the posterior midgut, respectively, along the anterio-posterior axis of the insect body. The malpighian tubules which ensure the osmoregulatory and excretory functions connect to the gut at the junction between the midgut and the hindgut. larval feature, and are major sites of digestion and absorption in other insects. ${ }^{[53]}$

The functioning of the digestive tract, with its resident commensal bacteria, is directly threatened by exogenous microbial pathogens. Consequently, gut epithelia are heavily shielded to resist microbial aggression by means of specialized junctions that seal the single cell layer, a thin cuticle that covers the foregut and the hindgut, and the PM which isolates the bolus from the midgut epithelial cells. ${ }^{[17]}$ As revealed by electron microscopy, four different layers of chitin fibrils and glycoproteins (e.g. peritrophins) ${ }^{[54,55]}$ are secreted at the beginning of the anterior midgut by the proventriculus ${ }^{[56]}$ and self-assemble to form the PM. ${ }^{[56]}$ Peritrophin genes are also expressed in more distal parts of the midgut, suggesting that this barrier is remodeled along the length of the gut. ${ }^{[40]}$

Studies using corrosive agents reveal a role for the PM in the defense against enteric bacteria both in Aedes and Drosophila. ${ }^{[57,58]}$ The chitin-binding protein drosocrystallin is strongly expressed upon oral infection, and drosocrystallin mutants show reduced PM width and a shorter lifespan. These mutants show increased susceptibility to oral infections by Serratia marsescens and Pseudomonas entomophila, or to ingestion of the Pseudomonas pore-forming toxin monalysin. These results demonstrate that the PM plays an important role in the host defense by protecting the gut cells from intestinal pathogens and/or their toxins. ${ }^{[59,60]}$ Additional studies also suggest that the PM protects insects from xenobiotics such as dichloro-diphenyl-trichloroethane (DDT ${ }^{[61]}$ ). In addition to the PM, a mucin layer covers the midgut enterocytes in Drosophila, but its role in the host defense has yet to be investigated. ${ }^{[17]}$

Physical barriers also play a critical role in preventing the deleterious effect of chronic activation of the immune response by the commensal microbes. ${ }^{[62]}$ Indeed, mutants for the big bang gene, which encodes multiple membrane-associated postsynaptic density protein 95 (PSD-95), discs large, zonula occludens-1 (ZO-1) (PDZ) domain containing protein isoforms, displayed loose septate junctions on the apical side of the enterocytes and a constitutive activation of the anterior midgut immune response. This phenotype correlates with a shortened lifespan of bbg mutants, which can be restored by clearing resident bacteria using antibiotic treatment. These observations are reminiscent of the chronic inflammation characteristic of mammalian bowel diseases and indicate that intact intestinal cell junctions are required for immune tolerance towards the endogenous gut microbes. The role of epithelial integrity in the host defense is further supported by the phenotype of MyoIB mutants. The myosin IB protein is required to maintain the highly 
ordered structure and composition of the brush border layer in the larval midgut epithelium. Both MyoIB and bbg mutant flies are hypersensitive to enteric infections by pathogenic bacteria. ${ }^{[62,63]}$

\section{The active immune response of the gut}

\section{AMP production}

A major aspect of Drosophila host defense is the pathogen-induced expression of AMPs through the activation of nuclear factor kappa-light-chain-enhancer of activated B cells (NF-KB) transcription factor family members. Two signaling cascades, Toll and immune deficiency (IMD), regulate the systemic immune response, characterized by secretion of AMPs from the fat body cells into the hemocoel cavity of the insect. ${ }^{[64]}$ These pathways are activated via the detection of microbial elicitors by cognate pattern recognition receptors (PRRs) of the peptidoglycan (PGN) recognition protein (PGRP) and the Gram-negative binding protein (GNBP) families. ${ }^{[65-67]}$ The Lys-type PGNs of Gram-positive bacteria and $\beta$-glucans from fungal cell walls trigger the Toll pathway upon binding to a PRR complex including PGRP-SA, GNBP1, and PGRP-SD and the GNBP3 receptor, respectively. ${ }^{[68-75]}$ The meso-diaminopimelic acid (DAP)-type PGNs of Gram-negative bacteria and bacilli activate the IMD pathway through transmembrane PGRP-LC and/or secreted/cytosolic PGRP-LE receptors. ${ }^{[76-85]}$ AMP expression is also elicited by the sensing of danger signals such as secreted microbial proteases upstream of the Toll pathway. ${ }^{[68,86]}$ This dual sensing mechanism allows a graded Toll pathway activation threshold, matched to the severity of the infection. Danger sensing also avoids the mechanisms deployed by some pathogens to impede the detection of microbial moieties by PRRs. ${ }^{[68]}$

In the gut epithelium, the expression of AMP-encoding genes is typically regulated by the IMD pathway and bacterial PGN represents the only identified elicitor to date [Figure 2]. ${ }^{[69,77,87]}$ Bacterial recognition is regionalized along the length of the gut. PGRP-LE is the predominant intracellular receptor for monomeric PGN in the midgut. PGRP-LC acts mostly in the proventriculus and the hindgut and concomitantly with PGRP-LE in the ventriculus as a detector of extracellular monomeric and polymeric PGN. ${ }^{[15,85]}$ The dominant members of the Drosophila gut bacteria, Acetobacter and Lactobacilli spp., carry DAP-type PGNs and activate the IMD pathway at a basal level, which allows homeostatic relationship between this microbiota and the host immune response. ${ }^{[15]}$

Any dysregulation of the IMD pathway alters this balance. For example, upregulation of AMP gene expression induces intestinal dysbiosis, marked by the overgrowth of Gluconobacter morbifer, which is normally only a minor com- ponent of the natural gut microbial community. The resultant gut pathology is associated with a reduced lifespan. ${ }^{[23,31]}$ Several layers of control ensure a tight regulation of the IMD pathway in order to tolerate the commensal microbiota and fight pathogen infection when required [Figure 2]. The first layer involves negative regulators of the IMD pathway, some of which alter the initial steps of sensing and signaling processes by targeting either the bacterial elicitor or the host receptor. Catalytic members of the PGRP family have an amidase activity, related to the bacteriophage T7 lysozyme, that degrades PGN into non-immunostimulatory molecules ${ }^{[88]}$ Among these catalytic PGRPs, PGRP-LB has a predominant role in the modulation of the IMD pathway in the gut. ${ }^{[31,89]} P G R P-L B$ expression is activated by the microbiota and further enhanced upon infection. ${ }^{[15,23]}$ In the absence of infection, PGRP-LB mutant flies express high levels of AMP, compared to wild-type flies. This increased expression is suppressed under axenic conditions, demonstrating that PGRP-LB is required to maintain the low basal immune response to microbiota. ${ }^{[31]}$ A non-catalytic, membrane-bound signaling-deficient PGRP, PGRP-LF, also acts as a negative regulator of the IMD pathway. ${ }^{[00,91]}$ PGRP-LF has two PGN recognition domains and a high affinity for PGRP-LC when bound to the monomeric PGN of Gram-negative bacteria, tracheal cytotoxin (TCT). Thus, PGRP-LF hinders the assembly of the receptor complex upstream of IMD.$^{[92]}$ At the intracellular level, the accessibility of both PGRP-LE and PGRP-LC receptors to the downstream components of the cascade is antagonized by Pirk (Poor IMD Response upon Knock in), also referred to as PIMS (PGRP-LC-interacting inhibitor of IMD signaling) or Rudra. ${ }^{[28,93,94]}$ Indeed, Aggarwal et al., showed that the Pirk inhibitor interacts with PGRP-LC, PGRP-LE, and IMD, thus disrupting the signaling complex. Like PGRP-LB, Pirk is a critical component in the negative feedback loop that maintains a balanced IMD response following bacterial infection. ${ }^{[28]}$ Interestingly, flies lacking PGRP-LF have developmental defects that are attenuated when flies are reared on antibiotics. ${ }^{[90]}$ Similarly, PGRP-LB, Pirk double mutants have reduced lifespan that is improved when flies are maintained in axenic conditions. ${ }^{[31]}$ Clearly, these negative regulators prevent the spurious activation of the immune response by the fly indigenous microbes. Additional negative regulators of the IMD pathway have been identified, mainly acting via ubiquitylation and the degradation of intracellular components of the cascade. ${ }^{[17,95-102]}$ Altogether, these regulatory components downregulate the IMD cascade following immune stimuli, allowing a balanced AMP response to be re-established once the infection is cleared. ${ }^{[103,104]}$

Another layer of control is provided by the functional compartmentalization of the Drosophila gut. ${ }^{[15,23,105]}$ Although the IMD pathway responds to the endogenous 


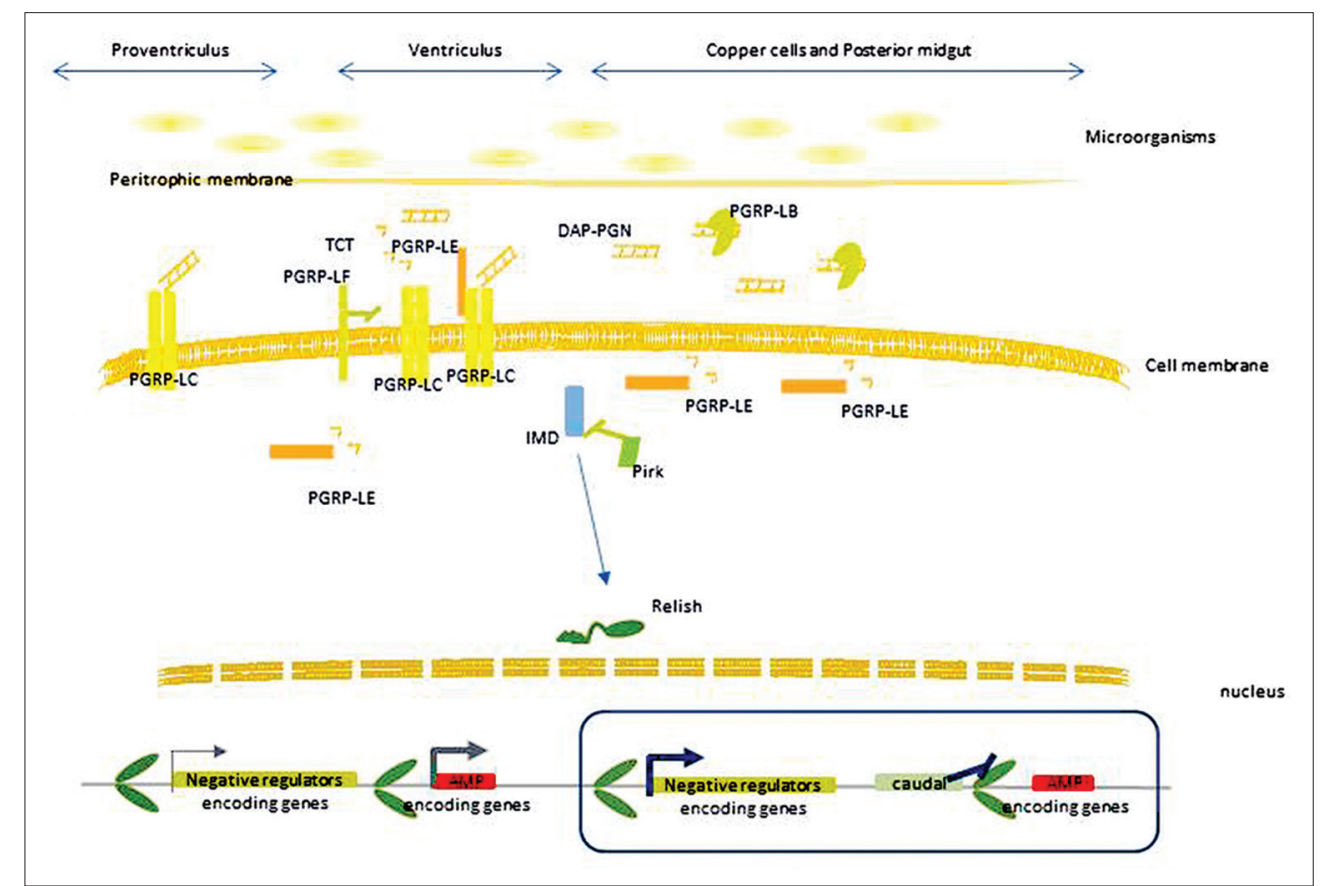

Figure 2: Regulation of the IMD pathway in the Drosophila midgut. The Drosophila gut is regionalized according to the major roles of PGRP receptors inducing the IMD pathway and to the nature of the concomitant induced responses. Monomeric and polymeric PGNs trigger the polymerization of PGRP-LC receptor isoforms which have a predominant role in the proventriculus. PGRP-LE exclusively controls the IMD pathway activity in the copper cells and the posterior midgut, where it acts as an intracellular receptor of TCT. Both receptors are required in the ventriculus. Following PGN binding, oligomerized receptors initiate an intracellular cascade involving the IMD adaptor protein, which leads to the nuclear translocation of a NF- $\mathrm{KB}$ transcription factor, Relish. Relish controls the expression of multiple genes including those encoding AMPs or negative regulators of the IMD pathway. Relish activity is inhibited by the homeobox gene caudal in the posterior section of the midgut. Signaling through the IMD pathway is modulated by the amidase activity of PGRP-LB which degrades PGN into non-stimulatory entities. The signaling complex of the IMD pathway is monitored by PGRP-LF which hinders the multimerization of the TCT-PGRP-LC complex and Pirk which limits the accessibility of the PGRP-LE and PGRP-LC receptors to the downstream components of the cascade. Filled arrows indicate the predominant response. Abbreviations used: AMPs: Antimicrobial peptides; NF-кB: Nuclear factor kappa-light-chain-enhancer of activated B cells; PGN: Peptidoglycan; PGRP: Peptidoglycan recognition protein; Pirk: Poor Imd Response upon Knock in; TCT: Tracheal cytotoxin.

microbes all along the gut, the expression of NF- $\mathrm{KB}-$ dependent AMPs and immunosuppressor genes follows different patterns. In the midgut, PGRP-LB and Pirk are constitutively expressed, whereas many AMP-encoding genes are repressed by the homeobox gene caudal [Figure 2]. ${ }^{[15,23]}$ Strikingly, the production of negative regulators, induced by the commensal bacterium Lactobacillus plantarum, is shifted toward PGRP-LE-mediated AMP production, in response to infection by Erwina carotovora. In 2012, Bosco-Drayon et al., proposed that AMP production might require stimulation by a higher bacterial load compared to negative regulator expression. This hypothesis is supported by the observation that IMD pathway activation increases with elevated microbiota density during aging. However, it is also tempting to speculate that a dual mechanism integrating the detection of PAMPs and danger signals would apply to the regulation of mucosal gut immunity, allowing the tolerance of autochthonous bacteria and directing the antimicrobial response toward allochthonous bacteria. Although such a mechanism has not been described for the IMD pathway to date, a transcriptomic analysis of the immune response in the gut of infected flies has revealed the induction of antimicrobial encoding genes, the drosomycin-like peptides, independently of the IMD pathway. These AMPs, proposed to be antifungal, are induced under stress conditions and intestinal damage through the activation of the Janus kinase-signal transducer and activator of transcription (JAK-STAT) pathway. ${ }^{[29,34]}$

\section{Reactive oxygen species production}

In addition to the orderly AMP production, a balanced redox system is essential for the host defense and maintenance of gut homeostasis in Drosophila. ${ }^{[106-109]}$ Oral infection of adult flies is associated with the rapid production of reactive oxygen species (ROS) ${ }^{[106,107]}$ generated by dual oxidase (DUOX), a member of the nicotinamide adenine dinucleotide phosphate (NADPH) oxidase family [Figure 3]. ${ }^{[106]}$ This ROS production is required to control dietary yeast, which would otherwise proliferate in the gut and kill the fly. ${ }^{[109]}$ Furthermore, RNA interference (RNAi)-mediated knock-down of duox in the intestinal epithelium leads to enhanced susceptibility of the flies to 


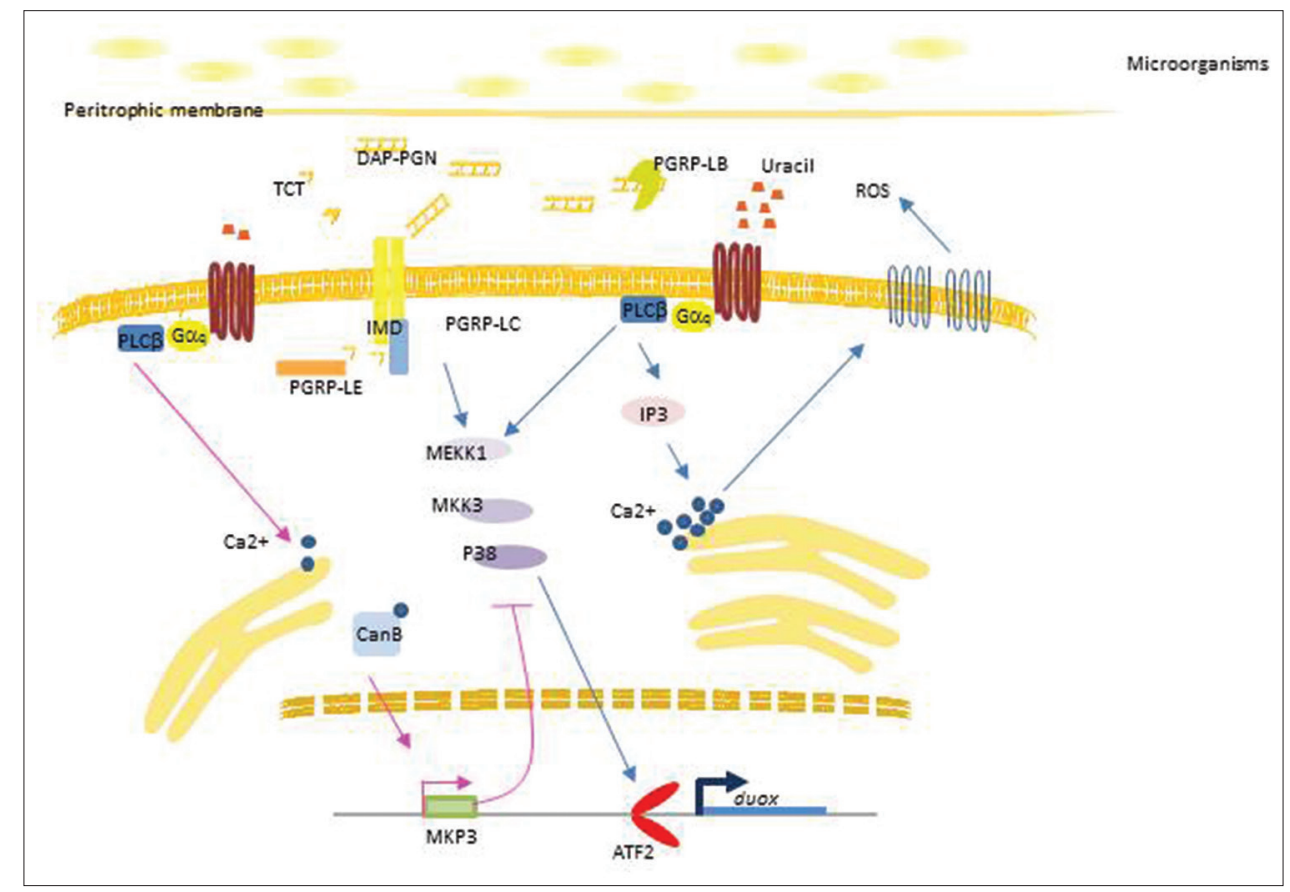

Figure 3: Regulation of ROS production in the Drosophila midgut. ROS are produced in the Drosophila gut lumen though the activity of DUOX. DUOX activity is triggered to a basal level by the gut microbiota and the ingested microorganisms, and is further enhanced upon infection. Uracil released by microorganisms is likely to trigger a G-protein-coupled receptor that activates the Goq-phospholipase $\mathrm{C} \beta$, which in turn mobilizes intracellular Ca2 + through IP3 generation for DUOX-dependent ROS production. DUOX activity is also regulated at the transcriptional level through the activation of the p38-MAPK pathway leading to the activation of the transcription factor Atf2. Transcription of the duox gene also integrates inputs from the Imd pathway. Under basal conditions, duox transcription is negatively controlled by the calcium-dependent phosphatase calcineurin B through the induction of mitogen kinase phosphatase 3. Blue and purple arrows indicate the enhancer and inhibitory pathways of DUOX activation, respectively. Abbreviations used: Duox: Dual oxidase; IP3: Inositol triphosphate; MAPK: Mitogen activated protein kinase; MEKK1: MAPKinase and ERK kinase kinase 1; MKK3: Mitogen activated protein kinase 3; ROS: Reactive oxygen species.

minor oral infections, demonstrating that DUOX is part of an essential defense system of the gut mucosa ${ }^{[106]}$ However, the oxidative burst is deleterious to the host cells and excess ROS are eliminated by the immune-regulated catalase (IRC), which is constitutively expressed in the gut. ${ }^{[107,109,110]}$ Both expression and activity of DUOX are enhanced upon infection [Figure 3]. The DUOX enzyme is calcium-dependent and is regulated by the Goq-phospholipase $\mathrm{C} \beta$ that mobilizes $\mathrm{Ca} 2+$ from the endoplasmic reticulum (RE) upon generation of inositol triphosphate. ${ }^{[106,109]}$ DUOX expression is regulated through the activation of the mitogen-activated protein (MAP) kinase pathway, integrating inputs from the PGRP-LC receptor but also from the sensing of microbial activities through a so far unidentified receptor acting upstream of PLC- $\beta$ [Figure 3]. ${ }^{[30,108]}$ The current model proposes that uracil produced by allochthonous bacteria is the determining agonist activating PLC- $\beta$, since uracil ingestion leads to ROS generation in a dose-dependent manner. This model is supported by the fact that oral infection with an uracil auxotrophic strain of $E$. carotovora shows increased virulence, while the auxotroph fails to trigger ROS production in the gut. ${ }^{[30]}$ However, the relevance of this observation remains uncertain, since the ability of the major components of the indigenous microbiota to produce uracil has not been demonstrated.

\section{Conclusion}

During the last 5 years, our comprehension of the mechanisms underlying the gut immune response has considerably expanded. The setting up of several infection models combined with genome-wide analyses revealed that the innate immune response in the gut is multilayered and tightly controlled to prevent immune reactions against the endogenous gut microbes. Similarly, the mechanisms involved in epithelial repair after exposure to microbial harm (i.e., resilience mechanisms) are now quite well understood. ${ }^{[50]}$

\section{From PAMP recognition to bacterial pathogenicity}

Several questions remain open

As both the subcellular distribution of PGRPs and the molecular nature of the uracil receptor are unknown, how the microbial PAMPs are sensed in the Drosophila 
gut remains unclear. In mice, the bacterial flagellin sensor Toll-like receptor 5 (TLR5) is located at the basal surface of enterocytes, but this sensor cannot detect flagellin from the gut luminal flora. Invasive bacteria, however, reach the basal enterocyte surface, where TLR5 is then able to trigger an inflammatory response. ${ }^{[111]}$ Septate junction deficient flies have an enhanced paracellular space that facilitates access of the endogenous flora to the latero-basal side of enterocytes. Similar to TLR5 distribution in mouse enterocytes, a basal cellular distribution of PGRP-LC may explain the potent innate immune response observed in these mutant flies. ${ }^{[62]}$

How the damage inflicted to the gut by pore-forming toxins, such as the Cry-toxins from Bacillus thuringiensis or monalysin from Pseudomonas entomophila, causes lethality in Drosophila is unclear. In mammals, any breach of the gut mucosal barrier results in basal exposure of TLR5 to flagellin from the endogenous flora, leading to TLR5 activation and subsequent chronic gut inflammation. ${ }^{[112]} \mathrm{A}$ similar mechanism in the fly would predict that if pore-forming toxins lead to internal exposure to the gut flora, then this exposure would result in enhanced IMD pathway activation and subsequent lethality ${ }^{[113]}$ Gut damage and food uptake blockage may also contribute to pore-forming toxin pathogenicity. ${ }^{[35]}$

\section{A chicken and egg conundrum, the aging gut phenotype}

The increased titer of the microbial community associated with enhanced IMD pathway activation during aging of the fly remains mysterious. ${ }^{[24]}$ Possibly, this paradoxical correlation could reflect a long-term selection of the bacteria for resistance, leading to a gradual increase in the basal response. Alternatively, increased IMD pathway activation could be linked to an age-related deterioration in gut repair mechanisms, leading to increased exposure to bacterial PAMPs and selection of resistant bacterial strains.

Despite the differences between the mammalian intestine and the fly gut, many parallels between the two establish Drosophila as a powerful and fascinating model to decipher fundamental aspects of gut biology. Such knowledge will have implications for understanding the dynamic host/microbiota interactions in the healthy human gut and for the complex mechanisms of intestinal disease syndromes such as Crohn's. ${ }^{[62]}$

\section{Acknowledgments}

We thank Prof D. Gubb for critical reading of the manuscript. This work has been published under the framework of : the LABEX : ANR-10-LABX-0036_NETRNA and benefits from a funding from the state managed by the French National Research Agency as part of the Investments for the future program ; the Centre National de la Recherche Scientifique and the European Research Council Advanced
Grant (AdG_20090506 "Immudroso"; to Prof JM. Reichhart). Dr L. El-Chamy is supported by the UNESCO-L'Oreal for women in sciences program and the research council of the Saint-Joseph University of Beirut.

\section{REFERENCES}

1. Mulcahy H, Sibley CD, Surette MG, Lewenza S. Drosophila melanogaster as an animal model for the study of Pseudomonas aeruginosa biofilm infections in vivo. PLoS Pathog 2011;7:e1002299.

2. Basset A, Khush RS, Braun A, Gardan L, Boccard F, Hoffmann JA, et al. The phytopathogenic bacteria Erwinia carotovora infects Drosophila and activates an immune response. Proc Natl Acad Sci USA 2000;97:3376-81.

3. Vodovar N, Vinals M, Liehl P, Basset A, Degrouard J, Spellman P, et al. Drosophila host defense after oral infection by an entomopathogenic Pseudomonas species. Proc Natl Acad Sci USA 2005;102:11414-9.

4. Liehl P, Blight M, Vodovar N, Boccard F, Lemaitre B. Prevalence of local immune response against oral infection in a Drosophila/ Pseudomonas infection model. PLoS Pathog 2006;2:e56.

5. Nehme NT, Liégeois S, Kele B, Giammarinaro P, Pradel E, Hoffmann JA, et al. A model of bacterial intestinal infections in Drosophila melanogaster. PLoS Pathog 2007;3:e173.

6. Cox CR, Gilmore MS. Native microbial colonization of Drosophila melanogaster and its use as a model of Enterococcus faecalis pathogenesis. Infect Immun 2007;75:1565-76.

7. Limmer S, Quintin J, Hetru C, Ferrandon D. Virulence on the fly: Drosophila melanogaster as a model genetic organism to decipher host-pathogen interactions. Curr Drug Targets 2011;12:978-99.

8. Glittenberg MT, Kounatidis I, Christensen D, Kostov M, Kimber S, Roberts I, et al. Pathogen and host factors are needed to provoke a systemic host response to gastrointestinal infection of Drosophila larvae by Candida albicans. Dis Model Mech 2011;4:515-25.

9. Tepass U, Hartenstein V. Epithelium formation in the Drosophila midgut depends on the interaction of endoderm and mesoderm. Development 1994;120:579-90.

10. Micchelli CA, Perrimon N. Evidence that stem cells reside in the adult Drosophila midgut epithelium. Nature 2006;439:475-9.

11. Ohlstein B, Spradling A. The adult Drosophila posterior midgut is maintained by pluripotent stem cells. Nature 2006;439:470-4.

12. Ohlstein B, Spradling A. Multipotent Drosophila intestinal stem cells specify daughter cell fates by differential notch signaling. Science 2007;315:988-92.

13. Jiang H, Edgar BA. EGFR signaling regulates the proliferation of Drosophila adult midgut progenitors. Development 2009;136:483-93.

14. Mathur D, Bost A, Driver I, Ohlstein B. A transient niche regulates the specification of Drosophila intestinal stem cells. Science 2010;327:210-3.

15. Bosco-Drayon V, Poidevin M, Boneca IG, Narbonne-Reveau K, Royet J, Charroux B. Peptidoglycan sensing by the receptor PGRP-LE in the Drosophila gut induces immune responses to infectious bacteria and tolerance to microbiota. Cell Host Microbe 2012;12:153-65.

16. Buchon N, Osman D, David FP, Fang HY, Boquete JP, Deplancke B, et al. Morphological and molecular characterization of adult midgut compartmentalization in Drosophila. Cell Rep 2013;3:1725-38. 
17. Lemaitre B, Miguel-Aliaga I. The digestive tract of Drosophila melanogaster. Annu Rev Genet 2013;47:377-404.

18. Lehane MJ. Peritrophic matrix structure and function. Annu Rev Entomol 1997;42:525-50.

19. Lehane MJ, Billingsley PF. Biology of the Insect Midgut. Heidelberg, Ger: Springer; 1996.

20. Murakami R, Takashima S, Hamaguchi T. Developmental genetics of the Drosophila gut: Specification of primordia, subdivision and overt-differentiation. Cell Mol Biol (Noisy-le-grand) 1999;45:661-76.

21. Chandler JA, Lang JM, Bhatnagar S, Eisen JA, Kopp A. Bacterial communities of diverse Drosophila species: Ecological context of a host-microbe model system. PLoS Genet 2011;7:e1002272.

22. Broderick NA, Lemaitre B. Gut-associated microbes of Drosophila melanogaster. Gut Microbes 2012;3:307-21.

23. Ryu JH, Kim SH, Lee HY, Bai JY, Nam YD, Bae JW, et al. Innate immune homeostasis by the homeobox gene caudal and commensal-gut mutualism in Drosophila. Science 2008;319:777-82.

24. Ren C, Webster P, Finkel SE, Tower J. Increased internal and external bacterial load during Drosophila aging without life-span trade-off. Cell Metab 2007;6:144-52

25. Corby-Harris V, Pontaroli AC, Shimkets LJ, Bennetzen JL, Habel KE, Promislow DE. Geographical distribution and diversity of bacteria associated with natural populations of Drosophila melanogaster. Appl Environ Microbiol 2007;73:3470-9.

26. Wong CN, Ng P, Douglas AE. Low-diversity bacterial community in the gut of the fruitfly Drosophila melanogaster. Environ Microbiol 2011;13:1889-900.

27. Bakula M. The persistence of a microbial flora during postembryogenesis of Drosophila melanogaster. J Invertebr Pathol 1969;14:365-74.

28. Lhocine N, Ribeiro PS, Buchon N, Wepf A, Wilson R, Tenev T, et al. PIMS modulates immune tolerance by negatively regulating Drosophila innate immune signaling. Cell Host Microbe 2008;4:147-58.

29. Buchon N, Broderick NA, Chakrabarti S, Lemaitre B. Invasive and indigenous microbiota impact intestinal stem cell activity through multiple pathways in Drosophila. Genes Dev 2009;23:2333-44.

30. Lee KA, Kim SH, Kim EK, Ha EM, You H, Kim B, et al. Bacterial-derived uracil as a modulator of mucosal immunity and gut-microbe homeostasis in Drosophila. Cell 2013;153:797-811.

31. Paredes JC, Welchman DP, Poidevin M, Lemaitre B. Negative regulation by amidase PGRPs shapes the Drosophila antibacterial response and protects the fly from innocuous infection. Immunity 2011;35:770-9.

32. Osman D, Buchon N, Chakrabarti S, Huang YT, Su WC, Poidevin M, et al. Autocrine and paracrine unpaired signaling regulate intestinal stem cell maintenance and division. J Cell Sci 2012;125:5944-9.

33. Gonzalez MR, Bischofberger M, Pernot L, van der Goot FG, Frêche B. Bacterial pore-forming toxins: The (w) hole story? Cell Mol Life Sci 2008;65:493-507.

34. Buchon N, Broderick NA, Poidevin M, Pradervand S, Lemaitre B. Drosophila intestinal response to bacterial infection: Activation of host defense and stem cell proliferation. Cell Host Microbe 2009;5:200-11.

35. Opota O, Vallet-Gely I, Vincentelli R, Kellenberger C, Iacovache I,
Gonzalez MR, et al. Monalysin, a novel ss-pore-forming toxin from the Drosophila pathogen Pseudomonas entomophila, contributes to host intestinal damage and lethality. PLoS Pathog 2011;7:e1002259.

36. Chakrabarti S, Liehl P, Buchon N, Lemaitre B. Infection-induced host translational blockage inhibits immune responses and epithelial renewal in the Drosophila gut. Cell Host Microbe 2012;12:60-70.

37. Jiang H, Patel PH, Kohlmaier A, Grenley MO, McEwen DG, Edgar BA. Cytokine/Jak/Stat signaling mediates regeneration and homeostasis in the Drosophila midgut. Cell 2009;137:1343-55.

38. Cronin SJ, Nehme NT, Limmer S, Liegeois S, Pospisilik JA, Schramek D, et al. Genome-wide RNAi screen identifies genes involved in intestinal pathogenic bacterial infection. Science 2009;325:340-3

39. Buchon N, Broderick NA, Kuraishi T, Lemaitre B. Drosophila EGFR pathway coordinates stem cell proliferation and gut remodeling following infection. BMC Biol 2010;8:152.

40. Buchon N, Broderick NA, Lemaitre B. Gut homeostasis in a microbial world: Insights from Drosophila melanogaster. Nat Rev Microbiol 2013;11:615-26

41. Shin SC, Kim SH, You H, Kim B, Kim AC, Lee KA, et al. Drosophila microbiome modulates host developmental and metabolic homeostasis via insulin signaling. Science 2011;334:670-4.

42. Sharon G, Segal D, Ringo JM, Hefetz A, Zilber-Rosenberg I, Rosenberg E. Commensal bacteria play a role in mating preference of Drosophila melanogaster. Proc Natl Acad Sci USA 2010;107:20051-6.

43. Storelli G, Defaye A, Erkosar B, Hols P, Royet J, Leulier F. Lactobacillus plantarum promotes Drosophila systemic growth by modulating hormonal signals through TOR-dependent nutrient sensing. Cell Metab 2011;14:403-14.

44. Ridley EV, Wong AC, Westmiller S, Douglas AE. Impact of the resident microbiota on the nutritional phenotype of Drosophila melanogaster. PLoS One 2012;7:e36765.

45. Choi NH, Kim JG, Yang DJ, Kim YS, Yoo MA. Age-related changes in Drosophila midgut are associated with PVF2, a PDGF/VEGF-like growth factor. Aging Cell 2008;7:318-34.

46. Biteau B, Hochmuth CE, Jasper H. JNK activity in somatic stem cells causes loss of tissue homeostasis in the aging Drosophila gut Cell Stem Cell 2008;3:442-55.

47. Rera M, Clark RI, Walker DW. Intestinal barrier dysfunction links metabolic and inflammatory markers of aging to death in Drosophila. Proc Natl Acad Sci USA 2012;109:21528-33.

48. Shoham S, Levitz SM. The immune response to fungal infections. Br J Haematol 2005;129:569-82.

49. Cognigni P, Bailey AP, Miguel-Aliaga I. Enteric neurons and systemic signals couple nutritional and reproductive status with intestinal homeostasis. Cell Metab 2011;13:92-104.

50. Ferrandon D. The complementary facets of epithelial host defenses in the genetic model organism Drosophila melanogaster: From resistance to resilience. Curr Opin Immunol 2013;25:59-70.

51. Bou Aoun R, Hetru C, Troxler L, Doucet D, Ferrandon D, Matt N. Analysis of thioester-containing proteins during the innate immune response of Drosophila melanogaster. J Innate Immun 2011;3:52-64.

52. Gregg TG, McCrate A, Reveal G, Hall S, Rypstra AL. Insectivory and social digestion in Drosophila. Biochem Genet 1990;28:197-207.

53. Nation JL. Insect Physiology and Biochemistry. CRC Press; 2002. 
54. Hegedus D, Erlandson M, Gillott C, Toprak U. New insights into peritrophic matrix synthesis, architecture, and function. Annu Rev Entomol 2009;54:285-302.

55. Lehane MJ, Allingham PG, Weglicki P. Composition of the peritrophic matrix of the tsetse fly, Glossina morsitans morsitans. Cell Tissue Res 1996;283:375-84.

56. King DG. Cellular organization and peritrophic membrane formation in the cardia (proventriculus) of Drosophila melanogaster. J Morphol 1988; 196:253-82.

57. Edwards MJ, Jacobs-Lorena M. Permeability and disruption of the peritrophic matrix and caecal membrane from Aedes aegypti and Anopheles gambiae mosquito larvae. J Insect Physiol 2000;46:1313-1320.

58. Wang P, Granados RR. Calcofluor disrupts the midgut defense system in insects. Insect Biochem Mol Biol 2000;30:135-43.

59. Kuraishi T, Binggeli O, Opota O, Buchon N, Lemaitre B. Genetic evidence for a protective role of the peritrophic matrix against intestinal bacterial infection in Drosophila melanogaster. Proc Natl Acad Sci USA 2011;108:15966-71.

60. Hayakawa T, Shitomi Y, Miyamoto K, Hori H. GalNAc pretreatment inhibits trapping of Bacillus thuringiensis Cry1 Ac on the peritrophic membrane of Bombyx mori. FEBS Lett 2004;576:331-5.

61. Tellam RL. The Peritrophic Matrix. London: Chapman and Hall; 1996.

62. Bonnay F, Cohen-Berros E, Hoffmann M, Kim SY, Boulianne GL, Hoffmann JA, et al. Big bang gene modulates gut immune tolerance in Drosophila. Proc Natl Acad Sci USA 2013;110:2957-62.

63. Hegan PS, Mermall V, Tilney LG, Mooseker MS. Roles for Drosophila melanogaster myosin IB in maintenance of enterocyte brush-border structure and resistance to the bacterial pathogen Pseudomonas entomophila. Mol Biol Cell 2007;18:4625-36.

64. Ferrandon D, Imler JL, Hetru C, Hoffmann JA. The Drosophila systemic immune response: Sensing and signalling during bacterial and fungal infections. Nat Rev Immunol 2007;7:862-74.

65. Royet J. Drosophila melanogaster innate immunity: An emerging role for peptidoglycan recognition proteins in bacteria detection. Cell Mol Life Sci 2004;61:537-46.

66. Royet J. Infectious non-self recognition in invertebrates: Lessons from Drosophila and other insect models. Mol Immunol 2004;41:1063-75.

67. Kaneko T, Silverman N. Bacterial recognition and signalling by the Drosophila IMD pathway. Cell Microbiol 2005;7:461-9.

68. Gottar M, Gobert V, Matskevich AA, Reichhart JM, Wang C, Butt TM, et al. Dual detection of fungal infections in Drosophila via recognition of glucans and sensing of virulence factors. Cell 2006;127:1425-37.

69. Leulier F, Parquet C, Pili-Floury S, Ryu JH, Caroff M, Lee WJ, et al. The Drosophila immune system detects bacteria through specific peptidoglycan recognition. Nat Immunol 2003;4:478-84.

70. Michel T, Reichhart JM, Hoffmann JA, Royet J. Drosophila toll is activated by Gram-positive bacteria through a circulating peptidoglycan recognition protein. Nature 2001;414:756-9.

71. Gobert V, Gottar M, Matskevich AA, Rutschmann S, Royet J, Belvin M, et al. Dual activation of the Drosophila toll pathway by two pattern recognition receptors. Science 2003;302:2126-30.

72. Pili-Floury S, Leulier F, Takahashi K, Saigo K, Samain E, Ueda R, et al. In vivo RNA interference analysis reveals an unexpected role for GNBP1 in the defense against Gram-positive bacterial infection in Drosophila adults. J Biol Chem 2004;279:12848-53.

73. Bischoff V, Vignal C, Boneca IG, Michel T, Hoffmann JA, Royet J. Function of the Drosophila pattern-recognition receptor PGRP-SD in the detection of Gram-positive bacteria. Nat Immunol 2004;5:1175-80.

74. Wang L, Gilbert RJ, Atilano ML, Filipe SR, Gay NJ, Ligoxygakis P. Peptidoglycan recognition protein-SD provides versatility of receptor formation in Drosophila immunity. Proc Natl Acad Sci USA 2008;105:11881-6.

75. Wang L, Weber AN, Atilano ML, Filipe SR, Gay NJ, Ligoxygakis P. Sensing of Gram-positive bacteria in Drosophila: GNBP1 is needed to process and present peptidoglycan to PGRP-SA. EMBO J 2006;25:5005-14.

76. Stenbak CR, Ryu JH, Leulier F, Pili-Floury S, Parquet C, Hervé M, et al. Peptidoglycan molecular requirements allowing detection by the Drosophila immune deficiency pathway. J Immunol 2004;173:7339-48.

77. Kaneko T, Goldman WE, Mellroth P, Steiner H, Fukase K, Kusumoto $\mathrm{S}$, et al. Monomeric and polymeric gram-negative peptidoglycan but not purified LPS stimulate the Drosophila IMD pathway. Immunity 2004;20:637-49.

78. Choe KM, Werner T, Stöven S, Hultmark D, Anderson KV. Requirement for a peptidoglycan recognition protein (PGRP) in Relish activation and antibacterial immune responses in Drosophila. Science 2002;296:359-62.

79. Gottar M, Gobert V, Michel T, Belvin M, Duyk G, Hoffmann JA, et al. The Drosophila immune response against Gram-negative bacteria is mediated by a peptidoglycan recognition protein. Nature 2002;416:640-4.

80. Rämet M, Manfruelli P, Pearson A, Mathey-Prevot B, Ezekowitz RA. Functional genomic analysis of phagocytosis and identification of a Drosophila receptor for E. coli. Nature 2002;416:644-8.

81. Werner T, Borge-Renberg K, Mellroth P, Steiner H, Hultmark D. Functional diversity of the Drosophila PGRP-LC gene cluster in the response to lipopolysaccharide and peptidoglycan. J Biol Chem 2003;278:26319-22.

82. Takehana A, Yano T, Mita S, Kotani A, Oshima Y, Kurata S. Peptidoglycan recognition protein (PGRP)-LE and PGRP-LC act synergistically in Drosophila immunity. EMBO J 2004;23:4690-700.

83. Kaneko T, Yano T, Aggarwal K, Lim JH, Ueda K, Oshima Y, et al. PGRP-LC and PGRP-LE have essential yet distinct functions in the Drosophila immune response to monomeric DAP-type peptidoglycan. Nat Immunol 2006;7:715-23.

84. Kurata S. Extracellular and intracellular pathogen recognition by Drosophila PGRP-LE and PGRP-LC. Int Immunol 2010;22:143-8.

85. Neyen C, Poidevin M, Roussel A, Lemaitre B. Tissue- and ligand-specific sensing of gram-negative infection in Drosophila by PGRP-LC isoforms and PGRP-LE. J Immunol 2012;189:1886-97.

86. El Chamy L, Leclerc V, Caldelari I, Reichhart JM. Sensing of 'danger signals' and pathogen-associated molecular patterns defines binary signaling pathways 'upstream' of toll. Nat Immunol 2008;9:1165-70.

87. Tzou P, Ohresser S, Ferrandon D, Capovilla M, Reichhart JM, Lemaitre B, et al. Tissue-specific inducible expression of antimicrobial peptide genes in Drosophila surface epithelia. Immunity 2000;13:737-48. 
88. Royet J, Dziarski R. Peptidoglycan recognition proteins: Pleiotropic sensors and effectors of antimicrobial defences. Nat Rev Microbiol 2007;5:264-77.

89. Zaidman-Rémy A, Hervé M, Poidevin M, Pili-Floury S, Kim MS, Blanot D, et al. The Drosophila amidase PGRP-LB modulates the immune response to bacterial infection. Immunity 2006;24:463-73.

90. Maillet F, Bischoff V, Vignal C, Hoffmann J, Royet J. The Drosophila peptidoglycan recognition protein PGRP-LF blocks PGRP-LC and IMD/JNK pathway activation. Cell Host Microbe 2008;3:293-303.

91. Persson C, Oldenvi S, Steiner H. Peptidoglycan recognition protein LF: A negative regulator of Drosophila immunity. Insect Biochem Mol Biol 2007;37:1309-16.

92. Basbous N, Coste F, Leone P, Vincentelli R, Royet J, Kellenberger C, et al. The Drosophila peptidoglycan-recognition protein LF interacts with peptidoglycan-recognition protein LC to downregulate the Imd pathway. EMBO Rep 2011;12:327-33.

93. Kleino A, Myllymäki H, Kallio J, Vanha-aho LM, Oksanen K, Ulvila J, et al. Pirk is a negative regulator of the Drosophila Imd pathway. J Immunol 2008;180:5413-22.

94. Aggarwal K, Rus F, Vriesema-Magnuson C, Ertürk-Hasdemir D, Paquette N, Silverman N. Rudra interrupts receptor signaling complexes to negatively regulate the IMD pathway. PLoS Pathog 2008;4:e1000120

95. Foley E, O'Farrell PH. Functional dissection of an innate immune response by a genome-wide RNAi screen. PLoS Biol 2004;2:E203.

96. Kim M, Lee JH, Lee SY, Kim E, Chung J. Caspar, a suppressor of antibacterial immunity in Drosophila. Proc Natl Acad Sci USA 2006;103:16358-63.

97. Khush RS, Cornwell WD, Uram JN, Lemaitre B. A ubiquitin-proteasome pathway represses the Drosophila immune deficiency signaling cascade. Curr Biol 2002;12:1728-37.

98. Thevenon D, Engel E, Avet-Rochex A, Gottar M, Bergeret E, Tricoire H, et al. The Drosophila ubiquitin-specific protease dUSP36/ Scny targets IMD to prevent constitutive immune signaling. Cell Host Microbe 2009;6:309-20.

99. Fernando MD, Kounatidis I, Ligoxygakis P. Loss of Trabid, a new negative regulator of the Drosophila immune-deficiency pathway at the level of TAK1, reduces life span. PLoS Genet 2014;10:e1004117.

100. Tsuda M, Langmann C, Harden N, Aigaki T. The RING-finger scaffold protein Plenty of SH3s targets TAK1 to control immunity signalling in Drosophila. EMBO Rep 2005;6:1082-7.
101. Aparicio R, Neyen C, Lemaitre B, Busturia A. dRYBP contributes to the negative regulation of the Drosophila Imd pathway. PLoS One 2013;8:e62052.

102. Yagi Y, Lim YM, Tsuda L, Nishida Y. Fat facets induces polyubiquitination of Imd and inhibits the innate immune response in Drosophila. Genes Cells 2013;18:934-45.

103. Myllymäki H, Valanne S, Rämet M. The Drosophila imd signaling pathway. J Immunol 2014;192:3455-62.

104. Aggarwal K, Silverman N. Positive and negative regulation of the Drosophila immune response. BMB Rep 2008;41:267-77.

105. Ryu JH, Nam KB, Oh CT, Nam HJ, Kim SH, Yoon JH, et al. The homeobox gene Caudal regulates constitutive local expression of antimicrobial peptide genes in Drosophila epithelia. Mol Cell Biol 2004;24:172-85.

106. Ha EM, Oh CT, Bae YS, Lee WJ. A direct role for dual oxidase in Drosophila gut immunity. Science 2005;310:847-50.

107. Ha EM, Oh CT, Ryu JH, Bae YS, Kang SW, Jang IH, et al. An antioxidant system required for host protection against gut infection in Drosophila. Dev Cell 2005;8:125-32.

108. Ha EM, Lee KA, Seo YY, Kim SH, Lim JH, Oh BH, et al. Coordination of multiple dual oxidase-regulatory pathways in responses to commensal and infectious microbes in Drosophila gut. Nat Immunol 2009;10:949-57.

109. Ha EM, Lee KA, Park SH, Kim SH, Nam HJ, Lee HY, et al. Regulation of DUOX by the Galphaq-phospholipase Cbeta-Ca2+pathway in Drosophila gut immunity. Dev Cell 2009;16:386-97.

110. Ryu JH, Ha EM, Oh CT, Seol JH, Brey PT, Jin I, et al. An essential complementary role of NF-kappaB pathway to microbicidal oxidants in Drosophila gut immunity. EMBO J 2006;25:3693-701.

111. Gewirtz AT, Navas TA, Lyons S, Godowski PJ, Madara JL. Cutting edge: Bacterial flagellin activates basolaterally expressed TLR5 to induce epithelial proinflammatory gene expression. J Immunol 2001;167:1882-5.

112. Rhee SH, Im E, Riegler M, Kokkotou E, O'brien M, Pothoulakis C. Pathophysiological role of Toll-like receptor 5 engagement by bacterial flagellin in colonic inflammation. Proc Natl Acad Sci USA 2005;102:13610-5.

113. Broderick NA, Raffa KF, Handelsman J. Midgut bacteria required for Bacillus thuringiensis insecticidal activity. Proc Natl Acad Sci USA 2006;103:15196-9. 